\title{
Assigning Significance in Label-Free Quantitative Proteomics to Include Single-Peptide-Hit Proteins with Low Replicates
}

\author{
Qingbo Li ${ }^{1,2}$ \\ ${ }^{1}$ Center for Pharmaceutical Biotechnology, College of Pharmacy, University of Illinois at Chicago, Chicago, IL 60607, USA \\ ${ }^{2}$ Department of Microbiology and Immunology, College of Medicine, University of Illinois at Chicago, Chicago, IL 60612, USA
}

Correspondence should be addressed to Qingbo Li, qkli@uic.edu

Received 18 March 2010; Revised 20 May 2010; Accepted 19 June 2010

Academic Editor: Andrew J. Link

Copyright ( 2010 Qingbo Li. This is an open access article distributed under the Creative Commons Attribution License, which permits unrestricted use, distribution, and reproduction in any medium, provided the original work is properly cited.

\begin{abstract}
When sample replicates are limited in a label-free proteomics experiment, selecting differentially regulated proteins with an assignment of statistical significance remains difficult for proteins with a single-peptide hit or a small fold-change. This paper aims to address this issue. An important component of the approach employed here is to utilize the rule of Minimum number of Permuted Significant Pairings (MPSP) to reduce false positives. The MPSP rule generates permuted sample pairings from limited analytical replicates and simply requires that a differentially regulated protein can be selected only when it is found significant in designated number of permuted sample pairings. Both a power law global error model with a signal-to-noise ratio statistic (PLGEM-STN) and a constant fold-change threshold were initially used to select differentially regulated proteins. But both methods were found not stringent enough to control the false discovery rate to $5 \%$ in this study. On the other hand, the combination of the MPSP rule with either of these two methods significantly reduces false positives with little effect on the sensitivity to select differentially regulated proteins including those with a single-peptide hit or with a $<2$-fold change.
\end{abstract}

\section{Introduction}

The increasing use of liquid chromatography/mass spectrometry (LC/MS) instrumentation for proteomics studies at a large scale stimulates the development and improvement of data analysis tools. The precise retrieval of biological information from a large LC/MS dataset critically depends on algorithms for data interpretation, which remains a current bottleneck in the rapid advance of proteomics technology [1]. The quantitation of differentially regulated proteins represents a major type of proteomics application in biological studies. Protein quantitation with LC/MS data includes three conceptually different methods, that is, spectral counting, differential stable isotope labeling, and label-free LC/MS measurements by using extracted ion chromatographic intensities [2]. Due to the increased time and complexity of sample preparation in stable isotope labeling, cost of labeling reagents and requirement of higher starting sample amount, however, researchers are increasingly using label-free proteomics for faster and simpler protein quantitation [3].
Multiple algorithms and software solutions for label-free proteomics data analysis have been developed [2]. These algorithms and software solutions provide quantitation of protein differential abundances but do not always provide a statistical significance assessment of differential abundances. Algorithms for statistical significance analysis in label-free proteomics with spectral counting were investigated $[4,5]$. In label-free quantitation with extracted ion chromatographic intensities, there are still needs to improve approaches for assessing statistical significance, especially for low-replicate datasets [6].

Most proteomics studies infer proteins with $\geq 2$ identified peptides as reliable protein identifications and usually disregard proteins with a single-peptide hit as unreliable for quantitation. This "two-peptide" rule was recently challenged with the evidence that it reduced protein identifications more in a target database than in a decoy database, and thus increased false discovery rates in protein identification [7]. Indeed, it was shown that proteins with a single-peptide hit could represent $30 \%$ of the proteins identified with $\geq 2$ MS2 spectrum matches at $P<.01$ [6]. Because those 
single-peptide proteins had $\geq 2$ MS2 spectrum matches $(P<$ .01 ) in multiple LC/MS analyses under the same condition, they had an adequate level of statistical confidence to be included for quantitation.

But the inclusion of single-peptide proteins in a differential quantitative proteomics analysis raises two issues. The first is that a conventional statistical test such as a $t$-test can not be applied toward these single-peptide proteins when the $t$-test relies on multiple quantified peptides as replicates to calculate the $t$-statistic for the protein relative abundance [6]. The second is that many single-peptide proteins are at a lower abundance and thus noisy. More stringent thresholds are needed to control the false discovery rate when these single-peptide proteins are included for the selection of differentially regulated proteins.

Pavelka et al. applied a power law global error model (PLGEM) and the signal-to-noise ratio (STN) statistic [8] to select differentially regulated proteins based on a spectral counting quantitation method [4]. The PLGEMSTN statistic utilized a resampling approach to estimate the null distribution from replicates of a sample. After the error model was calculated from a pool of resampling statistics that constituted the null distribution, a set of STN thresholds were applied at a specified confidence level toward samples with any level of replicates. The PLGEM-STN method is attractive in that it could be applied toward samples with no replicates if several replicates for one sample are provided to estimate the null distribution. It is also applicable to proteins with any number of identified peptides. The PLGEMSTN method, however, has not been demonstrated for label-free quantitation with extracted ion chromatographic intensities.

In this paper, the PLGEM-STN statistic was applied toward a LC/MS dataset obtained with a high-resolution mass spectrometer [9]. The peptide and protein abundances were quantified with a label-free approach based on extracted ion chromatographic intensities [6]. The false discovery rate was estimated at different confidence levels of the PLGEM-STN statistics. The PLGEM-STN statistic alone did not provide a desired level of false discovery rate control. Insufficient stringency in false discovery rate control was similar to the situation when a $t$-test statistic was used alone [6]. With the combination of a $t$-test and the rule of Minimum number of Permuted Significant Pairings (MPSP), however, the false discovery rate was significantly reduced in that study [6].

In this study, the combination of MPSP and PLGEMSTN was tested for controlling the false discovery rate in order to extend the selection of differentially regulated proteins to those with lower fold-changes and to those with single-peptide hits. The combination of MPSP and foldchange thresholds was also compared with the PLGEM-STNMPSP approach.

\section{Materials and Methods}

2.1. Cell Cultures and Proteins Samples. The Mycobacterium smegmatis $(\mathrm{Msm})$ strain $\mathrm{mc}^{2} 155$ was obtained from the
American Type Culture Collection (ATCC; Rockville, Md) and cultured in 7H9 media [10]. A pH 5.0 and a pH 7.0 Msm culture were grown in triplicate in unlabeled media and harvested as described previously $[6,9]$. A cell pellet was collected from a 30-ml culture aliquot for each culture replicate in a $\log$ phase. A $\left[{ }^{15} \mathrm{~N}\right]$-labeled Msm culture was also grown for use as a control to determine false positive rates in protein quantitation [10]. Hereafter, the Stressed $\mathrm{pH}$ 5 culture is named as $\mathbf{S}$, the Reference $\mathrm{pH} 7$ culture as $\mathbf{R}$, and the Control culture as $\mathbf{C}$.

As described previously [10], the medium for growing ${ }^{15} \mathrm{~N}$ labeled cells consisted of $(\mathrm{g} / \mathrm{L}) 99 \mathrm{At} \%\left({ }^{15} \mathrm{NH}_{4}\right)_{2} \mathrm{SO}_{4}: 0.5$; glucose: 2; Tween 80: 0.5; citric acid: 0.094; biotin: 0.0005; pyridoxine: $0.001 ; \mathrm{NaCl}: 0.1 ; \mathrm{Na}_{2} \mathrm{HPO}_{4}$ : $2.5 ; \mathrm{KH}_{2} \mathrm{PO}_{4}$ : 1 ; $\mathrm{MgSO}_{4} \cdot 6 \mathrm{H}_{2} \mathrm{O}: 0.1 ; \mathrm{CuSO}_{4} \cdot 5 \mathrm{H}_{2} \mathrm{O}: 0.001 ; \mathrm{ZnSO}_{4} \cdot 6 \mathrm{H}_{2} \mathrm{O}$ : 0.002; $\mathrm{CaCl}_{2} \cdot 2 \mathrm{H}_{2} \mathrm{O}: 0.0007$; ferric ammonium citrate: 0.04 ; $\mathrm{pH}$ 5.0. The single ${ }^{15} \mathrm{~N}$ labeled cell culture was grown at $50 \mathrm{ml}$ in a loosely capped $250-\mathrm{ml}$ nephelo culture flask under shaking at $37^{\circ} \mathrm{C}$. Thirty milliliter of the ${ }^{15} \mathrm{~N}$ labeled reference culture was collected at OD 1.1 in the late-log phase.

2.2. Protein Sample Preparation. Preparation of proteins from the cell pellets of cultures $S, R$, and $C$ was described previously $[6,10]$. The $S$ triplicates were pooled to generate protein sample $S_{P}$ and the $R$ triplicates were pooled to generate protein sample $R_{P}[6]$. In addition, the $S$ triplicates $S_{A}, S_{B}$, and $S_{C}$ were also individually processed. These five protein samples; that is, $S_{P}, R_{P}, S_{A}, S_{B}$, and $S_{C}$ were, respectively, mixed with an equal amount of proteins from the $\left[{ }^{15} \mathrm{~N}\right]$-labeled $\mathrm{C}$ culture. After mixing with the labeled proteins from culture $\mathrm{C}$, the five protein samples were separated on a 1D-SDS/PAGE gel, divided into five fractions, and processed for in-gel digestion and peptide extraction for LC/MS analysis as described in $[9,10]$. For the pooled samples $S_{P}$ and $R_{P}$, all five fractions were analyzed by LC/MS. For $S_{A}, S_{B}$, and $S_{C}$, only the center fractions were analyzed by LC/MS.

2.3. Peptide Analysis. The peptide extract from each gel fraction was constituted in $\sim 25 \mu \mathrm{l} 5 \%$ formic acid and was analyzed in duplicate injections with a nanoLC/LTQ-FTMS system (Thermo Finnigan; San Jose, CA) [6]. In each LC/MS injection, $5 \mu \mathrm{l}$ of peptide extract solution was separated on a $150-\mathrm{mm} \times 75-\mu \mathrm{m}$ C18 reverse phase column with $5 \%$ to $35 \%$ acetonitrile $(\mathrm{v} / \mathrm{v})$ gradient in $0.1 \%$ trifluoroacetic acid over 60 minutes. The LTQ-FTMS was operated in a data-dependent acquisition mode with up to $10 \mathrm{MS} / \mathrm{MS}$ spectra acquired following each MS scan. The acquired RAW data files were imported into BioWorks for peptide and protein identification. The BioWorks (Thermo Finnigan; San Jose, CA) software was on a stand-alone workstation and utilized Sequest as the search engine. The RAW data files were searched against an NCBI Msm database in two separate BioWorks searches. One search corresponded to $\left[{ }^{14} \mathrm{~N}\right]$-labeled peptides and proteins. The other corresponded to $\left[{ }^{15} \mathrm{~N}\right]$-labeled peptides and proteins. The precursor ion tolerance was set at $\pm 1.5 \mathrm{Da}$ to include the peptides, which precursor ions had one ${ }^{13} \mathrm{C}$ isotope. Trypsin was designated as the digestion enzyme with two allowed missed 
cleavages. Peptide and protein probabilities were calculated in BioWorks. Only the peptide charge states (PCSs) with $P<.01$ were accepted for subsequent quantitation. Lists of PCSs selected at $P<.01$ were exported from BioWorks into Excel spreadsheets. The Excel spreadsheets containing the accepted PCSs, along with RAW data files, were processed for quantitation as previously described [10-12]. The abundance of a PCS was represented by the extracted ion chromatographic intensity. The LC/MS raw data associated with this paper can be downloaded from http://proteomecommons.org/ Tranche (see supplementary material available online at doi:10.1155/2010/731582).

2.4. Protein Quantitation. Protein abundances were quantified with a label-free approach as described in $[6,9]$. The abundance of a protein was calculated as the sum of the extracted ion chromatographic intensities of the PCSs detected for that protein [9]. The unlabeled protein samples were named as $S_{A}, S_{B}, S_{C}, S_{P}$, and $R_{P}$. The $\left[{ }^{15} \mathrm{~N}\right]-$ labeled protein sample from culture $\mathrm{C}$ had five sample preparation replicates because it was mixed with each of the five unlabeled proteins samples. Accordingly, each sample preparation replicate of the culture $C$ protein sample was named by adding the prefix " $\mathrm{c}$ " before the unlabeled protein sample with which it was run together. For example, the labeled sample that was mixed with $S_{P}$ was named $c S_{P}$, and so forth. Thus, the labeled $\mathrm{C}$ culture protein sample had five replicates that were named as $c S_{A}, c S_{B}, c S_{C}, c S_{P}$, and $c R_{P}$, respectively. Because each sample was analyzed in duplicate LC/MS injections, the LC/MS injections were named by adding the subscript 1 or 2 to each protein sample (see Table 1).

Therefore, the LC/MS analysis of the five protein samples led to 20 quantitation categories (Table 1). Here, a quantitation category referred to one LC/MS injection of a protein sample in unlabeled or labeled form. Because each protein sample contained the unlabeled proteins from culture $\mathrm{S}$ or $\mathrm{R}$, and the labeled proteins from control culture C, one LC/MS injection generated four quantitation categories with two belonging to the unlabeled protein sample and two to the labeled protein sample. The five unlabeled protein samples $\left(S_{A}, S_{B}, S_{C}, S_{P}\right.$, and $\left.R_{P}\right)$, the five sample preparation replicates of the labeled control protein sample $\left(\mathrm{cS}_{\mathrm{A}}, \mathrm{cS}_{\mathrm{B}}, \mathrm{cS}_{\mathrm{C}}, \mathrm{cS} \mathrm{P}\right.$, and $\left(\mathrm{R}_{\mathrm{P}}\right.$ ), and the 20 quantitation categories arising from the duplicate analysis of these samples are summarized in Table 1.

2.5. Normalization among Sample Fractions. The complete analysis of the five gel fractions for $S_{P}$ and $R_{P}$ resulted in the quantitation of 5134 PCSs and 1032 proteins (see Tables 1 and 2 in Supplementary Material available online at doi:10.1155/2010/731582). In the label-free quantitation approach employed here, the abundance of a PCS ( $\left.A_{\mathrm{PCS}}\right)$ was represented by the extracted ion chromatographic intensity of the PCS, and the abundance of a protein $\left(A_{\mathrm{PRO}}\right)$ was represented by the sum of the extracted ion chromatographic intensities of the PCSs that belonged to the protein.
Because the sample fractionation efficiency might dictate the approach to normalize the samples, the fractionation resolution was examined by plotting a histogram for the percentage of the detected PCSs versus the number of gel fractions in which they were present (Figure 2). The result shows that $82.8 \%$ of the PCSs were present in a single gel fraction and $96.3 \%$ were present in $\leq 2$ gel fractions. Thus, a majority of PCSs were detected only in one gel fraction. These PCSs were called single-band PCSs.

The selection of the single-band PCSs was for the purpose of normalizing PCS abundances in different fractions [13]. In each fraction, the PCS abundances were normalized in the following two steps. In the first normalization step, the PCS abundances were normalized by the median extracted ion chromatographic intensity sought from the single-band PCSs. Then, the median-normalized PCSs intensities were multiplied by the total intensity of the same fraction averaged over all of the samples.

In these two steps of normalization, the first mediannormalization step improves the comparability of PCSs in each fraction across different samples. The second normalization step retained the relative fraction intensity information across the five fractions, so that the $A_{\mathrm{PCS}}$ values correlated more adequately to their protein abundances in the samples. This two-step normalization approach is depicted in Figure 3 as well.

It is critical to perform the second step of normalization because it preserves the information about the abundance of a protein in a sample. The information about the abundance of a protein in the samples will be indispensable to perform the power law global error and signal-to-noise statistic modeling as described later.

After PCS normalization, the protein abundance was calculated by summing the $A_{\mathrm{PCS}}$ values of that protein in each sample $[14,15]$.

\section{Results}

The purpose of this study was two-fold. One was to extend the selection of differentially regulated proteins to those that had single-peptide hits. The other was to select differentially regulated proteins at smaller fold-changes and at a false discovery rate $\leq .05$. The approaches to achieve this twofold purpose were investigated under a scenario where the number of sample replicates was too small to apply other typical statistics such as a $t$-test. More importantly, a conventional $t$-test alone might not provide the necessary specificity in the label-free quantitation of differentially regulated proteins. Therefore, in a prior test, it was found necessary to insert an additional measure, such as the MPSP rule [6].

The biological sample model used in the study was the proteome response of an acid stressed Msm culture (S) in reference to a neutral pH culture (R) [9]. Both $\mathrm{S}$ and $\mathrm{R}$ cultures were unlabeled. The proteins from a $\left[{ }^{15} \mathrm{~N}\right]$-labeled control culture (C) was used as an internal standard to mix with the proteins from the unlabeled cultures (Figure 1). Because the proteins from the control culture were analyzed repeatedly 
TABLE 1: The five unlabeled protein samples from culture $S$ or $R$, the five protein sample preparation replicates of the labeled proteins from culture $\mathrm{C}$, and the corresponding 20 quantitation categories (see Methods) ${ }^{\mathrm{a}}$.

\begin{tabular}{lcccccccccc}
\hline & \multicolumn{3}{c}{ Unlabeled protein sample from culture $\mathrm{S}$ or $\mathrm{R}$} & \multicolumn{5}{c}{$\left[{ }^{15} \mathrm{~N}\right]$-labeled protein sample from control culture C } \\
& $\mathrm{S}_{\mathrm{P}}$ & $\mathrm{R}_{\mathrm{P}}$ & $\mathrm{S}_{\mathrm{A}}$ & $\mathrm{S}_{\mathrm{B}}$ & $\mathrm{S}_{\mathrm{C}}$ & $\mathrm{cS}$ & $\mathrm{cR}_{\mathrm{P}}$ & $\mathrm{cS}_{\mathrm{A}}$ & $\mathrm{cS}_{\mathrm{B}}$ & $\mathrm{cS}_{\mathrm{C}}$ \\
\hline Quantitation & $\mathrm{S}_{\mathrm{P}, 1}$ & $\mathrm{R}_{\mathrm{P}, 1}$ & $\mathrm{~S}_{\mathrm{A}, 1}$ & $\mathrm{~S}_{\mathrm{B}, 1}$ & $\mathrm{~S}_{\mathrm{C}, 1}$ & $\mathrm{cS} \mathrm{S}_{\mathrm{P}, 1}$ & $\mathrm{cR}_{\mathrm{P}, 1}$ & $\mathrm{cS}_{\mathrm{A}, 1}$ & $\mathrm{cS}_{\mathrm{B}, 1}$ & $\mathrm{cS}_{\mathrm{C}, 1}$ \\
category & $\mathrm{S}_{\mathrm{P}, 2}$ & $\mathrm{R}_{\mathrm{P}, 2}$ & $\mathrm{~S}_{\mathrm{A}, 2}$ & $\mathrm{~S}_{\mathrm{B}, 2}$ & $\mathrm{~S}_{\mathrm{C}, 2}$ & $\mathrm{cS} \mathrm{S}_{\mathrm{P}, 2}$ & $\mathrm{cR}_{\mathrm{P}, 2}$ & $\mathrm{cS}_{\mathrm{A}, 2}$ & $\mathrm{cS}_{\mathrm{B}, 2}$ & $\mathrm{cS}_{\mathrm{C}, 2}$ \\
\hline
\end{tabular}

${ }^{a} S_{A}, S_{B}$, and $S_{C}$ were the unlabeled protein samples from the $S$ culture triplicates. $S_{P}$ was the pool of $S_{A}, S_{B}$, and $S_{C}$. R $R_{P}$ was the pool of the unlabeled proteins from the culture R triplicates. The subscripts " 1 " and " 2 " represent the LC/MS duplicates. Only the 3 rd gel fraction for samples $S_{A}, S_{B}$, and $S_{C}$ along with the labeled control in them was analyzed by LC/MS. All of the five gel fractions were analyzed with LC/MS for samples $S_{P}$ and $R_{P}$ along with the labeled control in them.

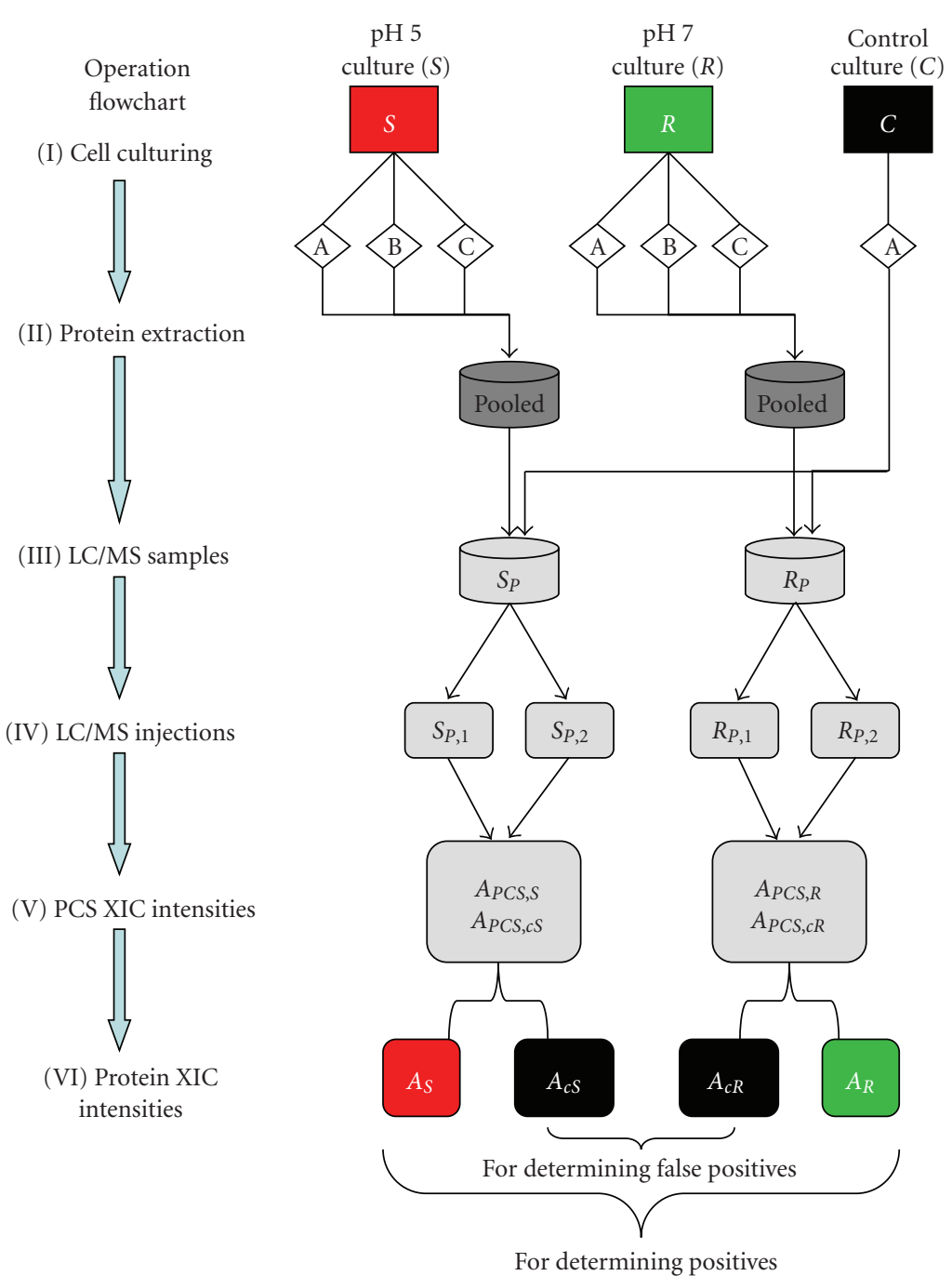

FIGURE 1: Experimental outline of the label-free protein quantitation approach to assess the acid stress response between the unlabeled stressed culture $(\mathrm{S})$ and the unlabeled reference culture $(\mathrm{R})$ with the $\left[{ }^{15} \mathrm{~N}\right]$-labeled culture as control $(\mathrm{C})$. The procedures are divided into six stages (I-VI). Briefly, equal amounts of protein extract from the $S$ culture triplicates were pooled. Equal amounts of protein extract from the $\mathrm{R}$ culture triplicates were also pooled. Into these two pooled unlabeled protein samples, an equal amount of protein extract from the $\mathrm{C}$ culture was added. This resulted in the two pooled samples; that is, $\mathrm{S}_{\mathrm{P}}$ and $\mathrm{R}_{\mathrm{P}}$. The proteins differentially expressed between the $\mathrm{S}$ and $\mathrm{R}$ cultures were determined based on comparison of the abundances of the unlabeled proteins that is, $A_{\mathrm{S}}$ and $A_{\mathrm{R}}$, between samples $\mathrm{S}_{\mathrm{P}}$ and $\mathrm{R}_{\mathrm{P}}$. For the purpose of false discovery rate assessment, the abundances of the $\left[{ }^{15} \mathrm{~N}\right]$-labeled proteins that is, $A_{\mathrm{cS}}$ and $A_{\mathrm{cR}}$, were quantified and compared between $S_{\mathrm{P}}$ and $\mathrm{R}_{\mathrm{P}}$ in the same way as between $A_{\mathrm{S}}$ and $A_{\mathrm{R}}$. The proteins found differentially expressed between $A_{\mathrm{S}}$ and $A_{\mathrm{R}}$ were considered positives, because they reflected the difference between the $\mathrm{S}$ and $\mathrm{R}$ cultures. The proteins found differentially expressed between $A_{\mathrm{cS}}$ and $A_{\mathrm{cR}}$ in the labeled form were false positives, because difference was not expected from the identical C sample that was run concurrently with two unlabeled samples in separate runs. 


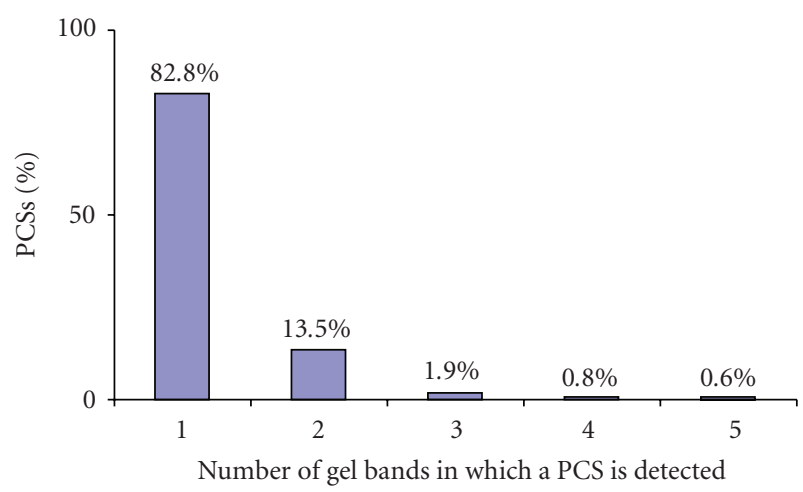

FIGURe 2: Histogram of the detected 5134 PCSs categorized based on the number of gel fractions in which they were present.

with two other unlabeled samples, the repeated analyses of the labeled control provided replicates to construct a null distribution in which no true differentially regulated proteins were present. The null distribution was used to derive an error model. Such an error model could not be derived from the pair of unlabeled protein samples $S_{P}$ and $R_{P}$ that did not have protein sample replicates.

With the null distribution provided by the labeled control sample, different approaches were experimented with to select differentially regulated proteins by using the combination of MPSP, PLGEM-STN, and fold-change. Differentially regulated proteins were selected from the unlabeled sample pair $S_{P}$ and $R_{P}$. The other three samples $S_{A}, S_{B}$, and $S_{C}$ were used to evaluate the source of variability but not for the selection of differentially regulated proteins. The naming of these samples and their LC/MS runs is delineated in Table 1.

This Results section consists of the following two subsections.

(1) Analyze the source of variability in the peptide and protein quantitation processes. An overview of this subsection is presented in Figure 4.

(2) Perform multistep extended selection of differentially regulated proteins. These steps are summarized in Table 2.

3.1. The Source of Variability in the Label-Free LC/MS Data. An observed differential abundance of a PCS or protein between samples arose not only from the difference in biological samples but also from measurement noise that included the variability among LC/MS injection replicates, sample preparation replicates, biological replicates, or the data processing method.

To assist in the assessment of the source of variability in the label-free quantitation of the LC/MS data, the 3rd of the five fractions of an SDS/PAGE gel lane was processed for LC/MS analysis for the protein samples $S_{A}, S_{B}, S_{C}, S_{P}$, and $R_{P}$ with duplicate injections for each sample (Table 1) [6]. The five samples with two LC/MS injections per sample resulted in $10 \mathrm{LC} / \mathrm{MS}$ runs. These $10 \mathrm{LC} / \mathrm{MS}$ runs of the 3 rd fraction allowed the quantitation of 349 proteins for the 3 rd fraction
[6]. Because a protein was quantified in both the unlabeled form (for culture $\mathrm{S}$ or $\mathrm{R}$ ) and the labeled form (for culture C), there were 20 quantitation categories for each protein. Thus, these 349 proteins and the 20 quantitation categories formed a $349 \times 20$ matrix. The $349 \times 20$ matrix was examined by a clustering analysis [16] to obtain an overview of the correlation among the protein samples and LC/MS injections with the purpose to reveal the major source of variability. The naming of the 20 quantitation categories was shown in Table 1.

From the clustering tree of the 20 quantitation categories shown in Figure 4, it could be seen that the distance between each pair of duplicate LC/MS injections was the shortest compared to those between any other sample pairings. The closest distance of the duplicate LC/MS injections for a sample indicated that the variability between LC/MS injections was the smallest, which also excluded that the label-free data analysis methodology [6] would introduce a significant variability.

In Figure 4, it was also apparent that the unlabeled and labeled quantitation categories were separated into two distinct branches represented by nodes I and II, respectively. The separation of the unlabeled and labeled quantitation categories into the two distinct clusters indicated that the difference between cultures $\mathrm{C}$ and $\mathrm{S}$ or $\mathrm{C}$ and $\mathrm{R}$ was larger than the difference between $S$ and R. From the tree branch under node II, it could be seen that the distance between the unlabeled protein samples $S_{P}$ and $R_{P}$ was larger than the distance among the $S$ culture replicates; that is, $S_{A}, S_{B}$, and $S_{C}$. The result indicated that the difference between cultures $S$ and $R$ exceeded the difference among the $S$ culture replicates, suggesting that the variability in biological sample replicates was less than the actual difference between the biological samples treated with different conditions.

Therefore, the clustering result in Figure 4 indicated that the variability increased in the order of LC/MS injections $<$ sample preparation replicates (under node I) biological replicates (under node III) < biological samples (between nodes III and IV). Because these differences were evaluated based on the proteomic quantitation data, a variability observed among biological replicates also included the variability introduced during sample preparation for LC/MS analysis. The similarity between the variability observed among the sample preparation replicates and the variability observed among the biological replicates suggested that the variability among biological replicates was not larger than the variability among sample preparation replicates.

\subsection{Extended Selection of Differentially Regulated Proteins.} This subsection describes the multiple steps leading to the extended selection of differentially regulated proteins from all quantified proteins including those with only a singlepeptide hit. The proteins with a single-peptide hit represent $1 / 3$ of the identified proteins. Therefore, it is desirable to have a procedure to select regulated proteins from all of the proteins including those with a single-peptide hit to maximize the potential of the global protein expression profiling. 


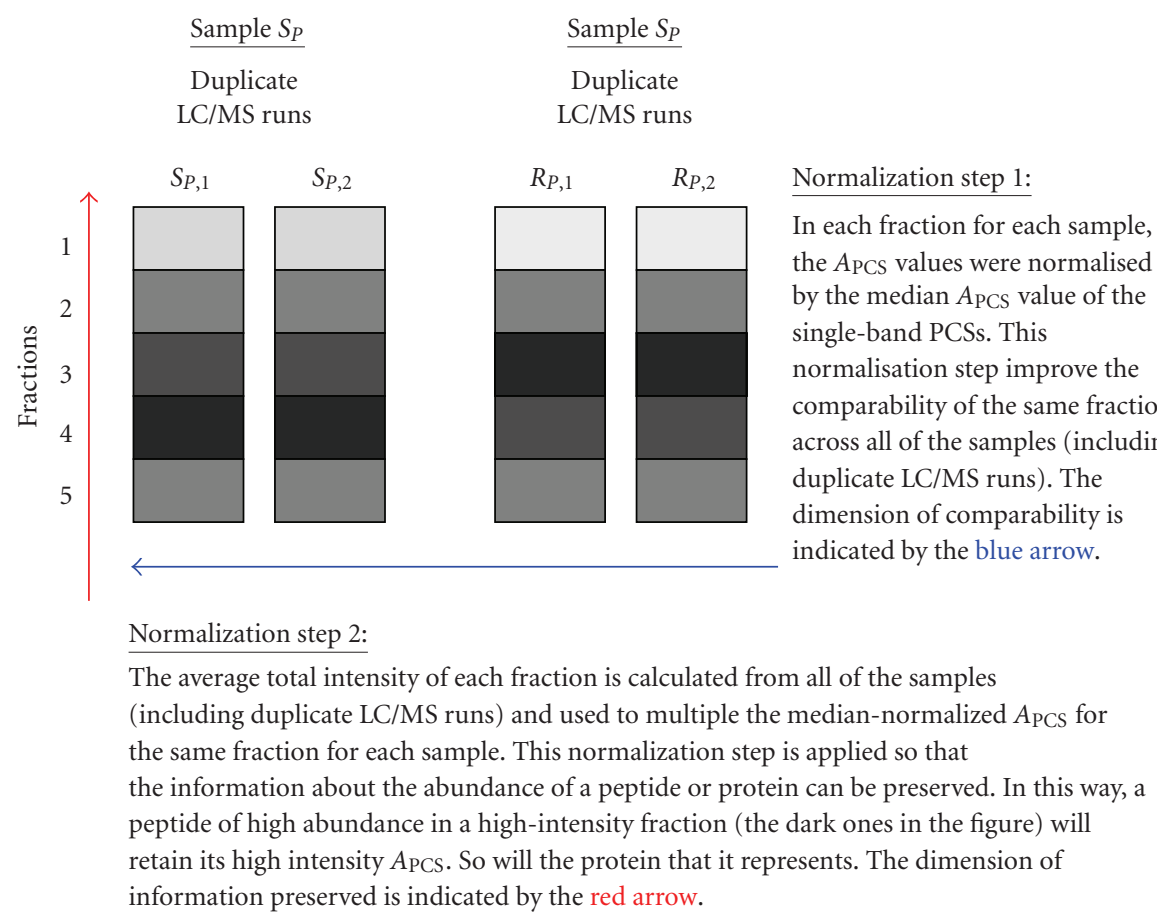

FIGURE 3: Schematic representation of the two-step normalization process. The gray boxes represent the fractions in the samples. Here, samples include the replicate LC/MS runs of a sample (see Figure 1 and Table 1 for the definitions). The shades indicate the hypothetical total peptide/protein intensities in the fractions. The degrees of darkness are for illustrative purpose and do not represent the actual experimental data.

The major steps to establish the criteria for extended selection of differentially regulated proteins are summarized in Table 2, and are described in detail in the following.

\subsubsection{The Null Distribution. Based on the evaluation with} the clustering analysis (Figure 4), the variability among sample preparation replicates appeared to be comparable with the variability among biological replicates. Samples $S_{P}$ and $R_{P}$ represented the average of triplicate biological replicates for cultures $S$ and $R$, respectively, because each of them was the pooled sample of three biological replicates. The pooling process further reduced the biological variability between $S_{P}$ and $R_{P}$. Therefore, the $\left[{ }^{15} \mathrm{~N}\right]$-labeled control sample replicates (Table 1) were adequate to represent a null distribution in which there was no differentially regulated protein.

The null distribution afforded an estimation of measurement noise. The determined measurement noise was then used to estimate the false discovery rate for the selected differentially regulated proteins between samples $S_{P}$ and $R_{P}$. The null distribution provided a reference for setting thresholds to maximize the selection of differentially regulated proteins (positives) while minimizing false positives. In Figure 5, such a null distribution was illustrated with the scatter plot represented by the pink dots.

To investigate the relationship between measurement variability and protein abundance $A_{\mathrm{PRO}}$, relative standard deviation (rSTD) was plotted against the mean $A_{\text {PRO }}$ value for each protein in the unlabeled protein samples (blue trace) or the labeled control protein samples (pink trace) (Figure 5). The rSTD-A $A_{\text {PRO }}$ trace in pink reflected the local noise of the null distribution. The local noise of the null distribution was mainly due to the variability that was introduced during sample preparation (Figure 4). The $\mathrm{rSTD}-A_{\mathrm{PRO}}$ trace in pink clearly suggested that the $A_{\mathrm{PRO}}$ measurement noise had a reciprocal dependence on the $A_{\mathrm{PRO}}$ amplitude. The rSTD- $A_{\mathrm{PRO}}$ trace in blue reflected both sample preparation variability and biological sample difference between cultures $\mathrm{S}$ and $\mathrm{R}$. Thus, the blue trace had higher rSTD values than the pink trace throughout the $A_{\text {PRO }}$ range.

\subsubsection{Modeling of Local Noise in the Null Distribution.} Because of the reciprocal dependence of $A_{\text {PRO }}$ rSTD on the

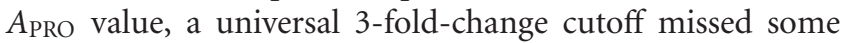
positives at higher $A_{\text {PRO values where a }<3 \text {-fold change was }}$ already significantly different from the local noise. Missed positives at higher $A_{\mathrm{PRO}}$ values could be observed in Figure 5 by examining the spread of the two scatter plots in the high $A_{\text {PRO }}$ ranges. At $A_{\mathrm{PRO}}>1000$, the rSTD was a few times smaller than that at $A_{\mathrm{PRO}}$ of $\sim 100$. From the figure, it could be seen that it was possible to detect a $<2$-fold change for the proteins with $A_{\mathrm{PRO}}>1000$. To the contrary, at $A_{\text {PRO }}<10$, a 3 -fold change threshold was not sufficient to eliminate many false positives. Therefore, a criterion adaptive to the dependence of $A_{\mathrm{PRO}}$ noise on $A_{\mathrm{PRO}}$ values would uncover more differentially regulated proteins. This extended selection of differentially regulated proteins could be achieved by penalizing proteins with higher $A_{\text {PRO }}$ values 


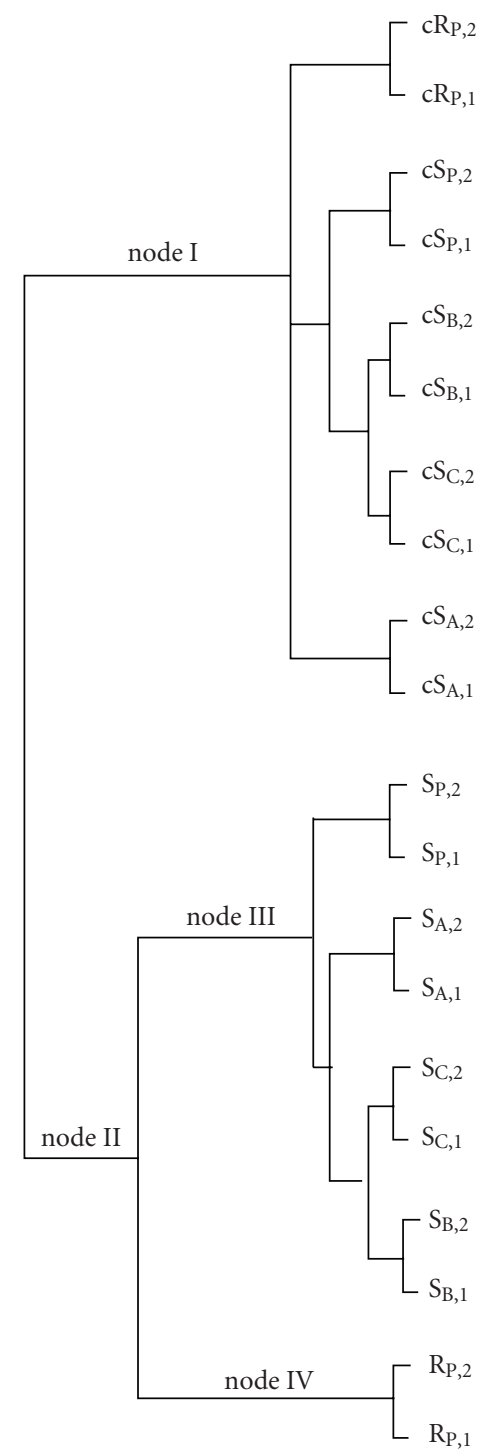

Figure 4: Clustering of the 20 quantitation categories based on the 349 proteins quantified from the 3 rd gel fraction for the five protein samples $S_{P}, R_{P}, S_{A}, S_{B}$, and $S_{C}$. Each protein sample contained the proteins from an unlabeled culture plus the labeled proteins from the control culture. Because a sample was run twice in LC/MS analysis, each protein sample had four quantitation categories of which two were for the unlabeled proteins and two for the labeled proteins. For example, $S_{P}$ had $S_{P, 1}$ and $S_{P, 2}$ for the unlabeled proteins from culture $S$ and $\mathrm{cS}_{\mathrm{P}, 1}$ and $\mathrm{cS}_{\mathrm{P}, 2}$ for the labeled proteins from culture C. The subscripts " 1 " and " 2 " indicate the duplicate LC/MS injections for a sample.

less than proteins with lower $A_{\mathrm{PRO}}$ values. Such an adaptive criterion, however, requires a systematic modeling of the noise to establish the thresholds according to local variability.

The issue of the dependence of variability on mean gene expression level was addressed for gene differential expression studies with DNA microarray. For example, Pavelka et al. proposed a power law global error model (PLGEM) [8] in combination with the signal-to-noise-ratio (STN) test statistic [17] for the identification of differentially expressed genes in microarray data. The PLGEM-STN approach estimated the null distribution by a resampling process. The approach could be applied to a varying number of replicates [8]. Pavelka et al. further applied the approach to spectral count-based quantitative proteomics data [4]. The PLGEMSTN statistic, however, has not been demonstrated for label- free proteomics data based on the quantitation of peptide and protein extracted ion chromatographic intensities.

In this study, the PLGEM-STN statistic was experimented with for the selection of differentially regulated proteins quantified with label-free proteomics based on protein extracted ion chromatographic intensities. The PLGEM-STN analysis was performed in four major steps for the dataset shown in Figure 5(see Scheme S1 in Supplementary Material available online at doi:10.1155/2010/731582). There were two reasons for the choice of the PLGEM-STN method. First, the PLGEM-STN method allowed statistical analyses of the proteins quantified with a single PCS because the PLGEMSTN statistic did not rely on multiple PCSs of a protein like a $t$-test [6]. Because single-peptide proteins constituted a third of the quantified proteins (Figure 6), being able to quantify 
TABLE 2: Overview of the key steps in extended selection of differentially regulated proteins.

\begin{tabular}{|c|c|c|c|}
\hline Step & Procedure & Justification & Utilized data \\
\hline 1 & $\begin{array}{l}\text { Establish a null } \\
\text { distribution }\end{array}$ & $\begin{array}{l}\text { A null distribution affords an estimation of measurement } \\
\text { noise originated from biological sample preparations and } \\
\text { analytical procedure. The noise will dictate the threshold } \\
\text { cutoff to distinguish regulated proteins from unregulated } \\
\text { ones. }\end{array}$ & $\begin{array}{l}\text { Protein abundances } A_{\mathrm{cS}} \text { and } \\
A_{\mathrm{cR}} \text { in the four quantitation } \\
\text { categories } \mathrm{cS}_{\mathrm{P}, 1}, \mathrm{cS}_{\mathrm{P}, 2}, \mathrm{cR}_{\mathrm{P}, 1} \text {, }\end{array}$ \\
\hline 2 & $\begin{array}{l}\text { Model local noise } \\
\text { in the null } \\
\text { distribution }\end{array}$ & $\begin{array}{l}\text { The measurement noise is not evenly distributed } \\
\text { throughout the range of different peptide and protein } \\
\text { abundances. Instead, the noise is locally dependent upon } \\
\text { the signal strength that is, peptide and protein } \\
\text { abundances, in a region. Thus, the threshold to select } \\
\text { regulated proteins could be different at different protein } \\
\text { abundance level. Modeling the distribution of noise } \\
\text { according to protein abundances will help to discern more } \\
\text { subtle changes for more abundant proteins while reduce } \\
\text { the false positives for less abundant proteins. }\end{array}$ & $\begin{array}{l}\text { and cR } \mathrm{R}_{\mathrm{P}, 2} \text { (Figure } 1 \text {; Table } 1 \text { ). } \\
\text { These four quantitation } \\
\text { categories represent the } \\
\text { replicate analyses of the same } \\
{\left[{ }^{15} \mathrm{~N}\right] \text {-labeled control protein }} \\
\text { sample run together with the } \\
\text { other two unlabeled protein } \\
\text { samples. Thus, regulated } \\
\text { proteins are not expected } \\
\text { from any pairing between } \\
\text { these four quantitation } \\
\text { categories. }\end{array}$ \\
\hline
\end{tabular}

PLGEM-STN statistic has been used in analyzing microarray data and spectral-count based quantitative proteomics data. The PLGEM approach establishes the distribution of noise according to gene/protein abundance level. In combination with STN statistic, adaptive thresholds are applied according to the protein abundance levels to maximize the selection of regulated proteins at higher abundance level while reduce the false positives for lower abundance proteins.

Due to the imperfection commonly found in many data sets and statistical models, the PLGEM-STN was not stringent enough to reduce false discovery rates in the label-free quantitative proteomics analysis. The MPSP rule is introduce to further reduce false discovery rates. The MPSP rule simply requires that a protein is accepted as a regulated one only if it is found regulated in multiple permutations of sample pairings using any kind of statistics, such as a $t$-test, PLGEM-STN, or even a fold-change threshold.

Select regulated proteins with the PLGEM-STNMPSP approach

The use of a combination of PLGEM-STN-MPSP approach reduces false discovery rates compared to PLGEM-STN statistic alone.

Select regulated proteins with a 6 fold-change-MPSP approach

Comparison of the PLGEM-STN-

MPSP and fold-change-MPSP approaches
The PLGEM-STN statistic over-penalizes the proteins with low abundances. A fold-change threshold in combination with MPSP is found more effective to select regulated proteins in the lower abundance region.

While the PLGEM-STN-MPSP approach over-penalizes lower-abundance proteins, the fold-change-MPSP approach over-penalizes the higher-abundance proteins. Thus, the two approaches are complimentary and can be used in combination.

\section{For determining false} positives: Use the protein abundances $A_{\mathrm{cS}}$ and $A_{\mathrm{cR}}$ in the four quantitation categories $\mathrm{cS}_{\mathrm{P}, 1}, \mathrm{cS}_{\mathrm{P}, 2}, \mathrm{cR}_{\mathrm{P}, 1}$, and $\mathrm{cR}_{\mathrm{P}, 2}$ (Figure 1; Table 1). For determining positives: Use the protein abundances $A_{\mathrm{S}}$ and $A_{\mathrm{R}}$ in the four quantitation categories $S_{\mathrm{P}, 1}$, $\mathrm{S}_{\mathrm{P}, 2}, \mathrm{R}_{\mathrm{P}, 1}$, and $\mathrm{R}_{\mathrm{P}, 2}$ (Figure 1; Table 1).

$\mathrm{S}_{\mathrm{P}, 1}$ and $\mathrm{S}_{\mathrm{P}, 2}$ represent the duplicate analyses of the unlabeled protein sample $S_{P}$ originated from the acid stressed culture $S . R_{P, 1}$ and $\mathrm{R}_{\mathrm{P}, 2}$ represent the duplicate analyses of the unlabeled protein sample $\mathrm{R}_{\mathrm{P}}$ originated from the reference neutral $\mathrm{pH}$ culture R. Thus, regulated proteins are expected from any pairings between these four quantitation categories that is, $\mathrm{S}_{\mathrm{P}, 1}, \mathrm{~S}_{\mathrm{P}, 2}, \mathrm{R}_{\mathrm{P}, 1}$, and $\mathrm{R}_{\mathrm{P}, 2}$. these single-peptide proteins was important to maximize the potential value of the data. Second, the PLGEM-STN method took into account the dependence of $A_{\mathrm{PRO}}$ noise on $A_{\mathrm{PRO}}$ levels. A threshold adjustable to the local dependence of $A_{\mathrm{PRO}}$ noise on $A_{\text {PRO levels allowed the selection of differentially }}$ regulated proteins with a smaller fold-change threshold at

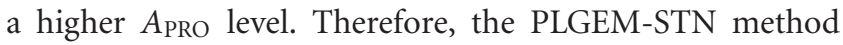
potentially could select more differentially regulated proteins 


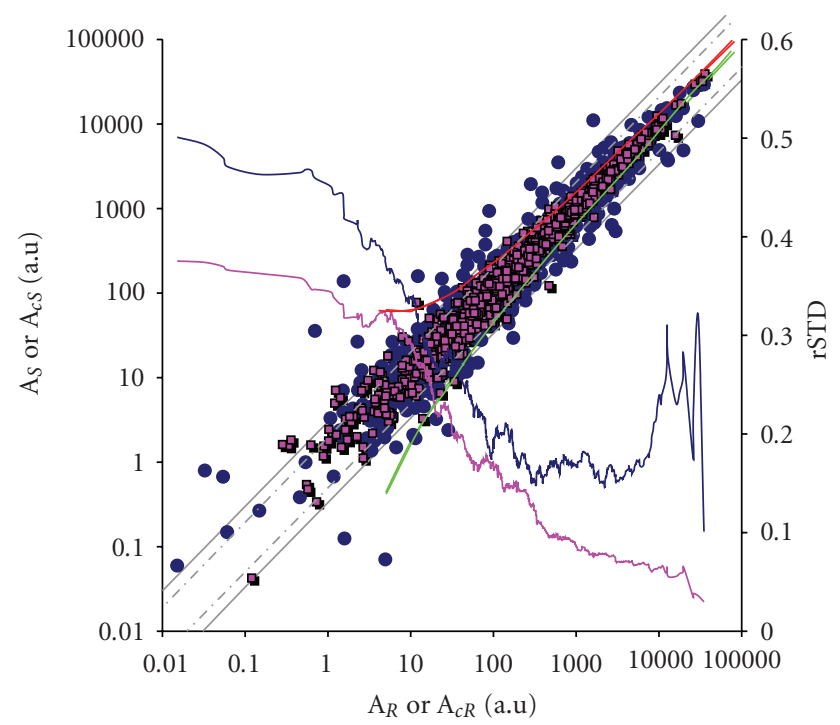

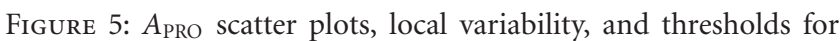
selecting differentially regulated proteins. The blue dots represent the $A_{\mathrm{PRO}}$ scatter plot of $A_{\mathrm{S}}$ versus $A_{\mathrm{R}}$ corresponding to the unlabeled proteins in sample $\mathrm{S}_{\mathrm{P}}$ versus $\mathrm{R}_{\mathrm{P}}$. $A_{\mathrm{S}}$ is the average of $A_{\mathrm{S}, 1}$ and $A_{\mathrm{S}, 2}$. $A_{\mathrm{R}}$ is the average of $A_{\mathrm{R}, 1}$ and $A_{\mathrm{R}, 2}$. The red dots represent the $A_{\mathrm{PRO}}$ scatter plot of $A_{\mathrm{cS}}$ versus $A_{\mathrm{cR}}$ corresponding to the labeled proteins in control sample replicate $\mathrm{cS}_{\mathrm{P}}$ versus $\mathrm{cR}_{\mathrm{P}} . A_{\mathrm{cS}}$ is the average of $A_{\mathrm{cS}, 1}$ and $A_{\mathrm{cS}, 2} . A_{\mathrm{cR}}$ is the average of $A_{\mathrm{cR}, 1}$ and $A_{\mathrm{cR}, 2} . A_{\mathrm{S}, 1}, A_{\mathrm{S}, 2}$, $A_{\mathrm{R}, 1}, A_{\mathrm{R}, 2}, A_{\mathrm{cS}, 1}, A_{\mathrm{cS}, 2}, A_{\mathrm{cR}, 1}$, and $A_{\mathrm{cR}, 2}$ were the $A_{\mathrm{PRO}}$ values for the eight quantitation categories defined in Table 1 . To evaluate the local noise of $A_{\text {PRO }}$ measurement, the relative standard deviation (rSTD) for each protein was calculated from its four unlabeled $A_{\text {PRO }}$ values $A_{\mathrm{S}, 1}, A_{\mathrm{S}, 2}, A_{\mathrm{R}, 1}$, and $A_{\mathrm{R}, 2}$ (the blue trace) or its four labeled $A_{\mathrm{PRO}}$ values $A_{\mathrm{cS}, 1}, A_{\mathrm{cS}, 2}, A_{\mathrm{cR}, 1}$, and $A_{\mathrm{cR}, 2}$ (the pink trace). The rSTD$A_{\mathrm{PRO}}$ traces were smoothed with a 100 -point moving box. The grey straight lines indicated a 3-fold (solid line) and a 2-fold (dashed line) change threshold. The solid red and green curves represent the fold-change thresholds established with the PLGEM-STN statistics based on the local variance in the null distribution (the pink-dot scatter plot).

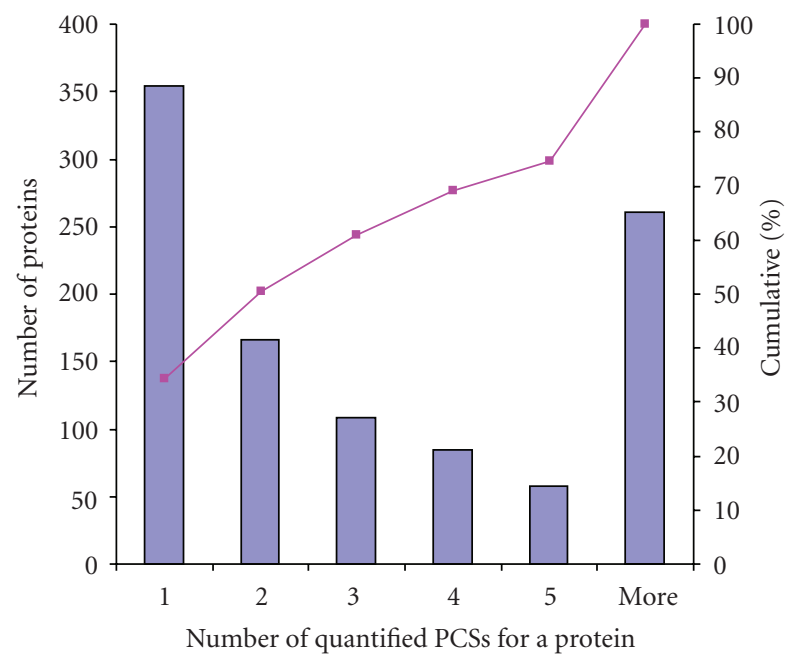

Figure 6: Histogram of PCS numbers for the 1032 quantified proteins. by applying a smaller fold-change threshold in the higher $A_{\text {PRO }}$ range where the variability was smaller. This possibility was tested as shown in the following.

3.2.3. Selection of Differentially Regulated Proteins with PLGEM-STN. Table 3 shows the result of the PLGEMSTN analysis for the unlabeled samples $S_{P}$ and $R_{P}$ and the labeled sample replicates $c S_{P}$ and $c R_{P} . c S_{P}$ and $c R_{P}$ were the labeled control samples analyzed concurrently with $S_{P}$ and $R_{P}$, respectively. The differentially regulated proteins found between $S_{P}$ and $R_{P}$ were positives, and those found between $c S_{p}$ and $\mathrm{cR}_{\mathrm{P}}$ were false positives. Because each protein sample was analyzed with duplicate LC/MS injections, permutation of the four LC/MS injections for a sample pair resulted in four permuted sample pairings [6]. These four permuted sample pairings were numbered as I to IV in Table 3. In each column for a permuted sample pairing in Table 3, the numbers of false positives and positives and the false discovery rate were listed. The false positives were determined as the differentially regulated proteins for the sample pair $c S_{p} / c_{p}$. The positives were determined as the differentially expressed proteins for the sample pair $\mathrm{S}_{\mathrm{P}} / \mathrm{R}_{\mathrm{P}}$. For the labeled protein sample pair $\mathrm{cS}_{\mathrm{P}} / \mathrm{CR}_{\mathrm{P}}$, the four permuted sample pairings were $c S_{\mathrm{P}, 1} / \mathrm{cR}_{\mathrm{P}, 1}, \mathrm{cS}_{\mathrm{P}, 1} / \mathrm{cR}_{\mathrm{P}, 2}$, $\mathrm{cS}_{\mathrm{P}, 2} / \mathrm{cR}_{\mathrm{P}, 1}$, and $\mathrm{cS}_{\mathrm{P}, 2} / \mathrm{cR}_{\mathrm{P}, 2}$. For the unlabeled sample pair $S_{\mathrm{P}} / R_{\mathrm{P}}$, the four permuted sample pairings were $S_{\mathrm{P}, 1} / R_{\mathrm{P}, 1}$, $\mathrm{S}_{\mathrm{P}, 1} / \mathrm{R}_{\mathrm{P}, 2}, \mathrm{~S}_{\mathrm{P}, 2} / \mathrm{R}_{\mathrm{P}, 1}$, and $\mathrm{S}_{\mathrm{P}, 2} / \mathrm{R}_{\mathrm{P}, 2}$. The naming of the LC/MS injections noted in the permuted sample pairings is shown in Table 1.

In Table 3, the positives and false positives were selected with the PLGEM-STN method at the confidence level of 0.01 and 0.002 , respectively. The results indicate that the numbers of positives or false positives were not the same among the four permuted sample pairings. To estimate an average false discovery rate, the numbers of positives and false positives were respectively averaged among the four permuted sample pairings. The false discovery rate was then calculated as the ratio of the average number of false positives divided by the average number of positives. The false discovery rate was determined at two different PLGEM-STN confidence levels (Table 3). With a receiver operating characteristic analysis, the PLGEM-STN approach is examined over a broader confidence level range (Figure 7) and will be compared with another approach that is to be described below.

3.2.4. Addition of the MPSP Rule. Initially, the PLGEM approach was carried out by comparing the duplicate LC/MS injections from the two samples $R$ and $S$ without permutation pairings. But the false discovery rate stayed high unless the sensitivity was severely compromised to reduce the false discovery rate. For example, at a confidence level of 0.0001 , only 16 differentially regulated proteins were selected at $6 \%$ false discovery rate (data not shown). With all of the permutation pairs and a combination of PLGEM and MPSP, 44 differentially regulated proteins were selected at a false discovery rate of 5\% (Table 3). Therefore, utilizing all possible permutation pairs with a combination of PLGEM and MPSP results in a higher sensitivity to uncover differentially regulated proteins. 
TABLE 3: Numbers of differentially regulated proteins selected with PLGEM-STN alone or in combination with MPSPa.

\begin{tabular}{|c|c|c|c|c|c|c|c|}
\hline \multirow{3}{*}{$\begin{array}{l}\text { PLGEM-STN } \\
\text { confidence level }\end{array}$} & \multirow{3}{*}{$\mathrm{FP}, \mathrm{P}$, and FDR } & \multicolumn{5}{|c|}{ PLGEM-STN } & \multirow{3}{*}{ PLGEM-STN-MPSP } \\
\hline & & \multicolumn{4}{|c|}{ Permuted sample pairings } & \multirow{2}{*}{ Average } & \\
\hline & & I & II & III & IV & & \\
\hline \multirow{3}{*}{0.01} & $\mathrm{FP}\left(\mathrm{cS}_{\mathrm{P}} / \mathrm{cR}_{\mathrm{P}}\right)$ & 31 & 68 & 22 & 46 & 42 & 13 \\
\hline & $\mathrm{P}\left(\mathrm{S}_{\mathrm{P}} / \mathrm{R}_{\mathrm{P}}\right)$ & 141 & 155 & 134 & 148 & 145 & 101 \\
\hline & FDR & 0.22 & 0.44 & 0.16 & 0.31 & 0.29 & 0.13 \\
\hline \multirow{3}{*}{0.002} & $\mathrm{FP}\left(\mathrm{cS}_{\mathrm{P}} / \mathrm{cR}_{\mathrm{P}}\right)$ & 6 & 15 & 3 & 9 & 8 & 2 \\
\hline & $\mathrm{P}\left(\mathrm{S}_{\mathrm{P}} / \mathrm{R}_{\mathrm{P}}\right)$ & 47 & 50 & 46 & 51 & 49 & 44 \\
\hline & FDR & 0.13 & 0.30 & 0.07 & 0.18 & 0.16 & 0.05 \\
\hline
\end{tabular}

${ }^{a}$ False positives (FP) were selected from sample pair $\mathrm{cS}_{\mathrm{P}} / \mathrm{cR}_{\mathrm{P}}$. Positives $(\mathrm{P})$ were selected from sample pair $\mathrm{S}_{\mathrm{P}} / \mathrm{R}_{\mathrm{P}}$. False discovery rate (FDR) was FP/P. The four permuted sample pairings (I-IV) were generated from the four LC/MS injections for a sample pair. See text.

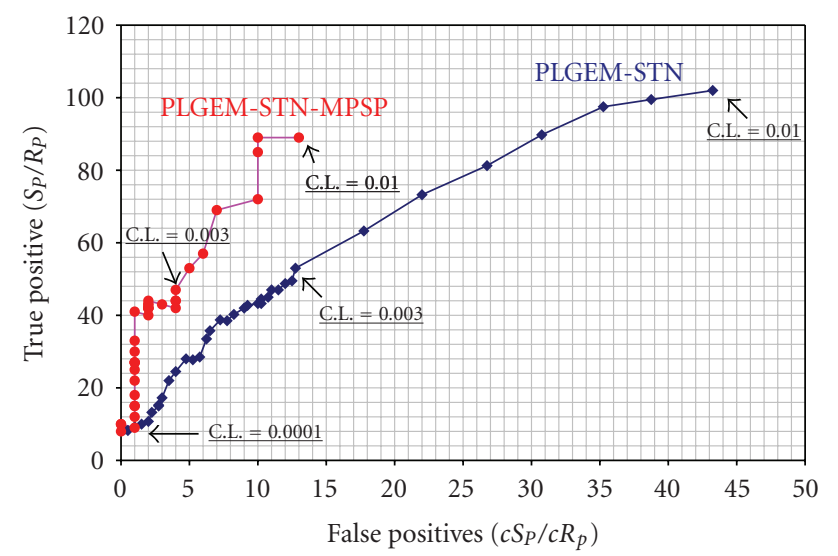

FIGURE 7: Receiver operating characteristic analysis of the PLGEMSTN approach with (red curve) or without (blue curve) the combination with MPSP. Positives are the differentially regulated proteins selected from the comparison of proteins abundances between samples $S_{\mathrm{P}}$ and $\mathrm{R}_{\mathrm{P}}$ (see Table 1 and Figure 1 for definition). False positives are the differentially regulated proteins selected from the comparison of proteins abundances between samples $\mathrm{cS}_{\mathrm{P}}$ and $\mathrm{cR}_{\mathrm{P}}$ (see Table 1 for definition). True positives are estimated by subtracting false positives from the positives. For each approach that is, PLGEM-STN-MPSP or PLGEM-STN, 37 data points at different confidence levels (C.L.) are plotted in this figure, starting from C.L. $=0.0001$ up to C.L. $=0.01$. The relationship between STN thresholds and C.L. can be found in Figure S2. The increment is 0.001 between C.L. of 0.0001 and 0.003 ( 30 data points). Between C.L. of 0.0 .003 and 0.01 , the increment is 0.01 ( 7 data points). The use of PLGEM here is similar to that in Table 3.

Because of the variable numbers of positives and false positives among the four permuted sample pairings, it was necessary to determine a consensus list of differentially regulated proteins from the four permuted sample pairings. Previously, the rule of MPSP was applied to determine the consensus list of differentially regulated proteins from four permuted sample pairings [6]. The MPSP rule required that only those proteins that were found differentially regulated in a certain number of permuted sample pairings were counted as positives (for $S_{P} / R_{P}$ ) or false positives (for $\mathrm{cS}_{\mathrm{P}} / \mathrm{cR}_{\mathrm{P}}$ ). When a sample pair such as $S_{\mathrm{P}} / \mathrm{R}_{\mathrm{P}}$ had no sample replicates but had duplicate LC/MS injections, MPSP was found to be optimum at four [6]. Setting MPSP at four meant that a differentially regulated protein had to be found differentially regulated in all of the four permuted sample pairings.

3.2.5. Selection of Differentially Regulated Proteins with the PLGEM-STN-MPSP Approach. The application of the MPSP rule towards the PLGEN-STN results decreased both false positives and positives (Table 3 ). But the false discovery rate was also decreased relative to that when only the PLGEMSTN statistic was applied. From Table 3, it could be seen that the number of true positives, which was estimated from the difference between the numbers of positives and false positives, remained about the same. Therefore, the combination of the MPSP rule with the PLEGM-STN method reduced the false discovery rate by $2-3$ times without compromising the sensitivity.

As summarized in Figure 7, the receiver operating characteristic analysis clearly shows that the PLGEM-STN-MPSP approach significantly reduces false positives to improve the specificity without significantly affecting the sensitivity. Compared to the use of the PLGEM-STN statistic alone, the combination of PLGEM-STN and MPSP performs better in controlling false discovery rates without compromising the sensitivity to select differentially regulated proteins.

\subsubsection{Selection of Differentially Regulated Proteins with a} Fold-Change-MPSP Approach. The use of MPSP with foldchange criteria was also examined (Table 4). With foldchange criteria alone, the false discovery rate did not drop below $46 \%$ at 2 - to 4 -fold changes (Table 4 ) or even at a 5fold change (See Figure S3 supplementary material available online at doi:10.1155/2010/731582). With the combination of MPSP and the fold-change criteria, the false discovery rate was reduced from $46 \%$ to $21 \%$ at 2 - and 3 -fold changes. At a 4 -fold change, the false discovery rate was reduced to $4 \%$. Compared to the combination of PLGEM-STN and MPSP, however, the combination of fold-change and MPSP reduced more true positives at the similar false discovery rate of $4 \%$ $5 \%$. Therefore, the application of MPSP with the fold-change criteria reduced sensitivity. The reduced sensitivity was due to the increase in the fold-change threshold.

With the 4-fold-change-MPSP and the PLGEM-STNMPSP approaches, 26 and 44 proteins were respectively 
TABLE 4: Numbers of differentially regulated proteins selected with fold-change threshold alone or in combination with MPSP.

\begin{tabular}{|c|c|c|c|c|c|c|c|}
\hline \multirow{3}{*}{ Fold change } & \multirow{3}{*}{ FP, $\mathrm{P}$, and FDR } & \multicolumn{5}{|c|}{ Fold-change } & \multirow{3}{*}{ Fold-change-MPSP } \\
\hline & & \multicolumn{4}{|c|}{ Permuted samplepairings } & \multirow{2}{*}{ Average } & \\
\hline & & I & II & III & IV & & \\
\hline \multirow{3}{*}{2} & $\mathrm{FP}\left(\mathrm{cS}_{\mathrm{P}} / \mathrm{cR}_{\mathrm{P}}\right)$ & 68 & 77 & 118 & 45 & 77 & 22 \\
\hline & $\mathrm{P}\left(\mathrm{S}_{\mathrm{P}} / \mathrm{R}_{\mathrm{P}}\right)$ & 171 & 154 & 186 & 147 & 165 & 104 \\
\hline & FDR & 0.40 & 0.50 & 0.63 & 0.31 & 0.47 & 0.21 \\
\hline \multirow{3}{*}{3} & $\mathrm{FP}\left(\mathrm{cS}_{\mathrm{P}} / \mathrm{cR}_{\mathrm{P}}\right)$ & 30 & 33 & 47 & 20 & 33 & 9 \\
\hline & $\mathrm{P}\left(\mathrm{S}_{\mathrm{P}} / \mathrm{R}_{\mathrm{P}}\right)$ & 66 & 70 & 85 & 60 & 70 & 42 \\
\hline & FDR & 0.45 & 0.47 & 0.55 & 0.33 & 0.47 & 0.21 \\
\hline \multirow{3}{*}{4} & $\mathrm{FP}\left(\mathrm{cS}_{\mathrm{P}} / \mathrm{cR}_{\mathrm{P}}\right)$ & 17 & 24 & 32 & 10 & 21 & 1 \\
\hline & $\mathrm{P}\left(\mathrm{S}_{\mathrm{P}} / \mathrm{R}_{\mathrm{P}}\right)$ & 42 & 50 & 53 & 35 & 45 & 26 \\
\hline & FDR & 0.40 & 0.48 & 0.60 & 0.29 & 0.47 & 0.04 \\
\hline
\end{tabular}

selected as differentially regulated at a false discovery rate of $4 \%$ or $5 \%$ (Tables 3 and 4 ). Among these 26 and 44 proteins, there were 55 unique proteins(see Table S1 in Supplementary Material available online at doi:10.1155/2010/731582). These 55 unique proteins included all of the 20 high-confidence differentially regulated proteins identified previously with an empirical fold-change and abundance level cutoff approach [9].

3.2.7. Comparison of the PLGEM-STN-MPSP and FoldChange-MPSP Approaches. Only 15 proteins were common between the two sets of differentially regulated proteins selected with the 4-fold-change-MPSP and the PLGEMSTN-MPSP approaches (Figure 8(a)). The 4-fold-changeMPSP approach selected more single-PCS proteins than the PLGEM-STN-MPSP approach (Figure 8(b)). The PLGEMSTN-MPSP approach selected proteins with a fold-change as low as 1.8 -fold (Figure 8(c)). However, these differentially regulated proteins selected with PLGEM-STN-MPSP had a protein abundance higher than most of the differentially regulated proteins selected with the 4-fold-change-MPSP approach (Figure $8(\mathrm{~d}))$. Thus, the two approaches complement each other and could be used simultaneously.

\section{Discussion}

4.1. Motivation of the Extensive Label-Free Quantitative Proteomics Analysis. Despite the relative complexity in label-free proteomics data analysis and the demand of more stringently controlled LC/MS experimental conditions, there are strong motivations stemming from biological and experimental perspectives to use the label-free approach, as discussed below.

As shown in Figure 4, the unlabeled and labeled quantitation categories are separated into two distinct clusters. One includes the quantitation categories from the labeled control culture C (under node I). The other includes the quantitation categories from the two unlabeled cultures $S$ and $\mathrm{R}$ (under node II). Thus, there was a larger difference between the labeled (C) and either of the two unlabeled samples ( $\mathrm{S}$ or $\mathrm{R}$ ) than between the two unlabeled cultures ( $\mathrm{S}$ and $\mathrm{R}$ ). The number of differentially regulated proteins between the labeled culture and either of the unlabeled culture was about three times as many as that between the two unlabeled cultures. Compared to the difference between the two unlabeled cultures, the difference between the labeled culture and either of the unlabeled cultures was larger. This larger difference was probably because the labeled culture was cultured in a synthetic minimal medium while the two unlabeled cultures were grown in a commercial 7H9 broth that was richer in ingredients. Another factor was that the acidic growth condition was a relatively mild stress so that not many proteins were differentially regulated.

The apparent difference in proteome profile for cells cultured in different media is actually a strong motivation for this study. In microbiological works, it is not always convenient to make a $\left[{ }^{15} \mathrm{~N}\right]$-labeled medium with complex ingredients required to cultivate bacteria under more physiologically relevant conditions. Even some of the stableisotope-labeled media are technically feasible to make, they often bear a costly price tag. For microbiological works, one might not want to be restricted by the type of medium that can be used because of the stable isotope labeling limitation. For example, some mycobacteria are difficult to cultivate on simple synthetic media and prefer complex media. Thus, unlabeled media are always convenient choices if the downstream proteomic analysis is established to proceed with the quantitation.

For such reasons, the focus of this study was on the comparison of protein expression profiles between the two unlabeled cultures $\mathrm{S}$ and R. The labeled control culture $\mathrm{C}$ was used as an internal standard to estimate false discovery rates.

4.2. The Use of a $\left[{ }^{15} \mathrm{~N}\right]$-Labeled Internal Standard for Null Distribution Construction in this Study. The label-free quantitation scheme presented in this study incorporated a labeled internal control to provide replicates for noise modeling without a requirement of other unlabeled sample replicates. The inclusion of a labeled internal control facilitates the control of false discovery rates. 


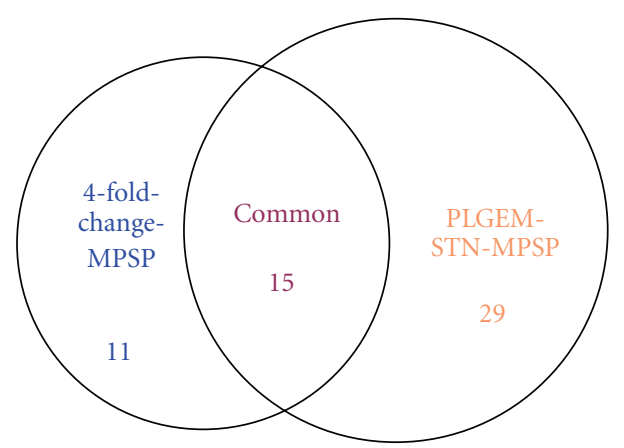

(a)

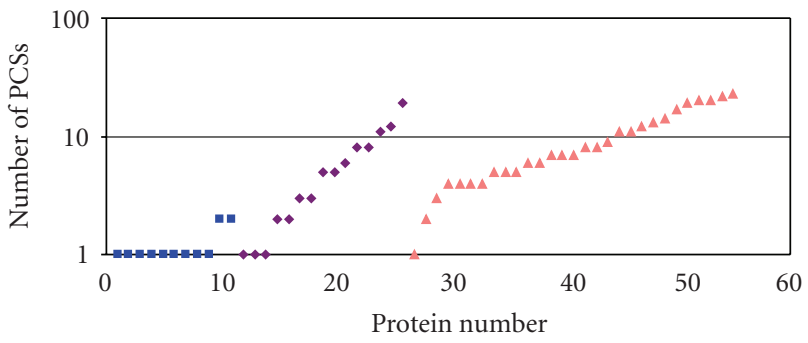

(b)

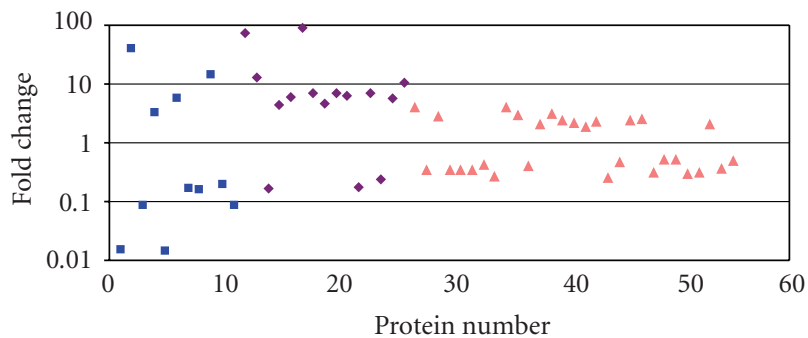

(c)

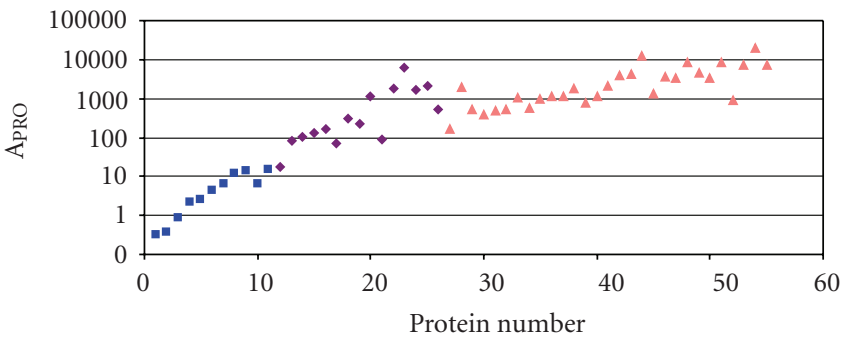

(d)

FIGURE 8: Comparison of the 26 and 44 differentially regulated proteins respectively selected by the 4-fold-change-MPSP and PLGEMSTN-MPSP approaches at a 5\% false discovery rate. (a) Overlap of the two sets of differentially regulated proteins. Panels (b-d) show the distributions of (b) the number of detected PCSs, (c) the fold changes, and (d) the abundances of the quantified proteins. The blue square, the purple diamond, and the tan triangle markers represent the differentially regulated proteins selected by 4 -fold-change-MPSP only, by both, and by PLGEM-STN-MPSP respectively. The protein number was from 1 to 55 on the $\mathrm{x}$-axis representing the 55 unique proteins ranked according to their $A_{\mathrm{PRO}}$ in each of the three groups (blue, purple, or tan).

Internal standards are commonly used to improve reliability of quantitative proteomics such as to aid in removing outlier data and to detect fluctuation in instrument performance [18].

Compared to other synthetic peptide internal standards $[18,19]$, the $\left[{ }^{15} \mathrm{~N}\right]$-labeled control culture $\mathrm{C}$ provides more comprehensive peptide internal standards. For most of the peptides, the extracted ion chromatographic intensities can be matched among the three protein samples originated from the two unlabeled ( $\mathrm{S}$ and $\mathrm{R}$ ) cultures and the labeled $(\mathrm{C})$ culture. The $\mathrm{C}$ protein sample was mixed and run together with either $\mathrm{S}$ or $\mathrm{R}$ protein sample, so that the reliability of the internal standards was improved. 
For constructing the null distribution for the error model in PLGEM-STN, it would be ideal to have the labeled internal standard identical to an unlabeled sample in protein composition. As mentioned above, however, that requirement could restrict the culturing conditions available for biological experiments. Thus, it is acceptable and sometimes necessary to use a labeled protein mixture sample as internal standard, even though the internal standard sample might be somewhat different from the unlabeled samples in protein abundance profiles.

Nevertheless, the null distribution is only utilized to establish the relation between the signal-to-noise ratio and the peptide abundance in the PLGEM-STN method. There is no requirement of direct one-to-one comparison between the labeled and unlabeled version of a protein during this process. Therefore, the difference in proteome composition between the labeled internal standard sample $\mathrm{C}$ and the two unlabeled samples $\mathrm{S}$ and $\mathrm{R}$ is not expected to affect the modeling parameters derived from the null distribution constructed from the labeled $\mathrm{C}$ sample.

One could choose to run multiple replicates of an unlabeled sample and use the replicates to construct the null distribution $[4,6]$. That approach would require more LC/MS runs as discussed previously [6].

\subsection{The Label-Free Data Analyses and Selection of Differ-} entially Regulated Proteins. The LC/MS data used in this work was acquired with a high-resolution mass spectrometer that resolved peptide peaks from a complex sample mixture to allow the determination of the extracted ion chromatographic intensities of peptides and proteins. Repeated LC/MS injections showed the highest reproducibility among several other types of replicates (Figure 4), indicating that the major variability of the label-free quantitation did not lie within the LC/MS separation and the data analysis method. Rather, sample preparation replicates represented a major source of the variability. With a labeled control sample to run concurrently with each of the unlabeled samples, replicates for the labeled control sample were obtained. The replicates of the control sample provided data to model the noise in the label-free quantitation with extracted ion chromatographic intensities (Figure 5).

We performed a two-step normalization procedure in which the information about the abundance of a peptide or protein in a sample was preserved (Figure 3). The preservation of the information about the abundance of a peptide or protein in the samples is critical for performing the PLGEM-STN analysis. In addition, because protein extracted ion chromatographic intensity was represented by the sum of the PCS extracted ion chromatographic intensities belonging to that protein, the summation weighed the low-intensity PCSs less than the high-intensity PCSs. Such a summation of PCS extracted ion chromatographic intensities probably suppressed noise from lower-intensity PCSs. When a protein abundance ratio is calculated as the average of PCS abundance ratios without weighing, the noise from a lower-intensity PCS would be amplified. We have avoided this potential issue by summing the PCS intensities to represent protein abundances before calculating protein abundance ratios.

Single-peptide proteins made up about $35 \%$ of the quantified proteins (Figure 6). Selection of differentially regulated proteins from these single-peptide proteins required a significance assessment method that did not rely on multiplepeptide detection to calculate a statistic about the confidence of a protein differential abundance. The use of a statistic that does not rely on the detection of multiple peptides is especially useful when the sample replicates are too low to use a typical statistical test such as a $t$-test. PLGEM-STN was a method that fits this criterion.

However, PLGEM-STN alone was not strict enough to control the false discovery rate without further diminishing the number of positives (Figure 7). The lack of stringency by using the PLGEM-STN method alone was similar to that by using the $t$-test alone [6]. In that prior study, the lack of specificity with a $t$-test alone was overcome by introducing the rule MPSP. The MPSP rule simply required that a protein be selected as differentially regulated only when it was repeatedly found so in certain number of permuted sample pairings. The MPSP rule was introduced to deal with datasets with small replicates where other more sophisticated statistical tests could not be applied [6]. Although the MPSP rule was originally used in combination with a $t$-test statistic and a fold-change threshold, this study shows that it can be used in combination with other types of statistical tests such as the PLGEM-STN method (Figure 7).

The combination of the MPSP rule allowed the selection of differentially regulated proteins at a false discovery rate $<5 \%$, which would have been impossible for a foldchange method, at least for the data used in this study (see Figure S3 supplementary material available online at doi:10.1155/2010/731582). The MPSP rule significantly reduced false positives while keeping the number of true positives relatively constant, thus effectively improving the statistical confidence of the selected differentially regulated proteins by lowering the false discovery rate (Table 4 ). The results from this and the prior study [6] suggest that MPSP is a rule that can be used in combination with different types of statistics to select differentially regulated proteins.

The label-free quantitation simplified cell culturing and sample preparation. Another useful aspect of the label-free quantitation is that peptide cross-reference could be used to increase the number of proteins quantified in all of the samples run under the same condition [13]. Lipton et al. [20] introduced the concept of accurate mass and elution time peptide tag for global protein quantitation using high resolution mass spectrometry. One advantage of this method over using the spectral counting method is that the large number of identifications that occur in a LC/MS injection can be used as the basis for improved quantitation of another LC/MS injection [13, 21, 22]. The accurate mass and elution time peptide tag approach uses the extracted ion chromatographic intensities as the quantitative measurement of peptides and proteins. The linear response of peptide extracted ion chromatographic intensities to protein quantities was demonstrated [23-25]. This method was thus 
used to improve the comparability of proteins quantified between samples, among LC/MS injections, and for different isotopic forms of a protein [14]. The quantitation of 349 proteins from a single gel fraction for several samples clearly demonstrated the power of the peptide cross-reference feature in extracted ion chromatographic intensity-based label-free quantitative proteomics [6].

One drawback of extracted ion chromatographic intensity-based label-free quantitative proteomics is that the success of an analysis critically depends upon the reproducibility of LC/MS runs that have to be maintained across multiple samples. The reproducibility of LC/MS runs across multiple samples is a prerequisite to reliable peptide cross-reference [13]. With the advancement in LC/MS instrumentation and the availability of improved LC/MS chromatogram alignment methods [26, 27], the reproducibility of LC/MS runs is unlikely to remain an obstacle for the increasing use of label-free quantitative proteomics.

\section{Conclusion}

A label-free quantitative proteomics scheme was demonstrated to select differentially regulated proteins with singlepeptide hits and with $<2$-fold changes at a $5 \%$ false discovery rate.

The label-free quantitation scheme incorporated a labeled internal control into multiple unlabeled samples to facilitate error modeling when there were no replicates for the unlabeled samples. The error modeling allowed the use of the PLGEM-STN statistic to facilitate the selection of differentially regulated proteins with single-peptide hits. The PLGEM-STN statistic also facilitated the selection of differentially regulated proteins at different fold-change thresholds according to the local abundance level of the proteins. While the PLGEM-STN statistic uncovered more differentially regulated proteins at higher abundance with smaller fold-changes, the PLGEM error modeling of local variance versus abundance overpenalized the proteins with lower abundance. With a constant fold-change threshold, however, differentially regulated proteins with higher abundance were overlooked. Thus, the results from this study showed that the PLGEM-STN and a constant fold-change threshold were complementary to each other and could be used simultaneously. But, neither the PLGEM-STN nor the 4 -fold-change criterion alone was stringent enough for selecting differentially regulated proteins at a $5 \%$ false discovery rate.

MPSP was introduced and shown to be a rule that could decrease false discovery rates when being used in combination with the PLGEM-STN statistic or the 4-foldchange threshold. The MPSP rule played a critical role in extending the selection of differentially regulated proteins to those with a single-peptide hit or with a lower foldchange in label-free proteomics when sample replicates were limited. Although the approaches were demonstrated for a representative replicate-limited scenario, they potentially can also be applicable to a situation where more sample replicates are available.

\section{Abbreviations}

PLGEM: Power Law Global Error Model

STN: Signal-To-Noise ratio

MPSP: Minimum number of Permuted Significant Pairings.

\section{Acknowledgments}

The nanoLC/LTQ-FTMS system and the BioWorks software utilized to acquire and process the data for this study were provided at the Proteomics and Informatics Services Facilities (PISF) at the Research Resources Center at University of Illinois at Chicago. The PISF was established by a grant from the Searle Funds at the Chicago Community Trust to the Chicago Biomedical Consortium. The author is indebted to the following individuals who contributed to the data used in this study. Bryan Roxas performed cell culturing, protein sample preparation, and BioWorks database search. Drs. Carrie Crot and Yan Wang performed LC/MS analysis of the peptide samples. The author also thanks Giovanni Lostumbo for help in proofreading the paper. Part of this work was supported by the NIH Grant R03AI073469-01A1.

\section{References}

[1] P. Mortensen, J. W. Gouw, J. V. Olsen et al., "MSQuant, an open source platform for mass spectrometry-based quantitative proteomics," Journal of Proteome Research, vol. 9, no. 1, pp. 393-403, 2010.

[2] L. N. Mueller, M.-Y. Brusniak, D. R. Mani, and R. Aebersold, "An assessment of software solutions for the analysis of mass spectrometry based quantitative proteomics data," Journal of Proteome Research, vol. 7, no. 1, pp. 51-61, 2008.

[3] W. Zhu, J. W. Smith, and C. M. Huang, "Mass spectrometrybased label-free quantitative proteomics," Journal of Biomedicine \& Biotechnology, vol. 2010, Article ID 840518, 6 pages, 2010.

[4] N. Pavelka, M. L. Fournier, S. K. Swanson et al., "Statistical similarities between transcriptomics and quantitative shotgun proteomics data," Molecular and Cellular Proteomics, vol. 7, no. 4, pp. 631-644, 2008.

[5] H. Choi, D. Fermin, and A. I. Nesvizhskii, "Significance analysis of spectral count data in label-free shotgun proteomics," Molecular and Cellular Proteomics, vol. 7, no. 12, pp. 23732385, 2008.

[6] Q. Li and B. A. P. Roxas, "An assessment of false discovery rates and statistical significance in label-free quantitative proteomics with combined filters," BMC Bioinformatics, vol. 10, article no. 43, 2009.

[7] N. Gupta and P. A. Pevzner, "False discovery rates of protein identifications: a strike against the two-peptide rule," Journal of Proteome Research, vol. 8, no. 9, pp. 4173-4181, 2009.

[8] N. Pavelka, M. Pelizzola, C. Vizzardelli et al., "A power law global error model for the identification of differentially expressed genes in microarray data," BMC Bioinformatics, vol. 5, article no. 203, 2004.

[9] B. A. P. Roxas and Q. Li, "Acid stress response of a mycobacterial proteome: insight from a gene ontology analysis," International Journal of Clinical and Experimental Medicine, vol. 2, no. 4, pp. 309-328, 2009. 
[10] B. A. P. Roxas and Q. Li, "Significance analysis of microarray for relative quantitation of LC/MS data in proteomics," BMC Bioinformatics, vol. 9, article no. 187, 2008.

[11] P. K. Rao, B. A. P. Roxas, and Q. Li, "Determination of global protein turnover in stressed mycobacterium cells using hybrid-linear ion trap-Fourier transform mass spectrometry," Analytical Chemistry, vol. 80, no. 2, pp. 396-406, 2008.

[12] V. P. Andreev, L. Li, T. Rejtar, Q. Li, J. G. Ferry, and B. L. Karger, "New algorithm for 15N//14N quantitation with LC-ESI-MS using an LTQ-FT mass spectrometer," Journal of Proteome Research, vol. 5, no. 8, pp. 2039-2045, 2006.

[13] V. P. Andreev, L. Li, L. Cao et al., "A new algorithm using cross-assignment for label-free quantitation with LC-LTQ-FT MS," Journal of Proteome Research, vol. 6, no. 6, pp. 2186-2194, 2007.

[14] P. K. Rao, G. Marcela Rodriguez, I. Smith, and Q. Li, "Protein dynamics in iron-starved Mycobacterium tuberculosis revealed by turnover and abundance measurement using hybrid-linear ion trap-fourier transform mass spectrometry," Analytical Chemistry, vol. 80, no. 18, pp. 6860-6869, 2008.

[15] P. K. Rao and Q. Li, "Principal component analysis of proteome dynamics in iron-starved mycobacterium tuberculosis," Journal of Proteomics and Bioinformatics, vol. 2, no. 1, pp. 1931, 2009.

[16] M. B. Eisen, P. T. Spellman, P. O. Brown, and D. Botstein, "Cluster analysis and display of genome-wide expression patterns," Proceedings of the National Academy of Sciences of the United States of America, vol. 95, no. 25, pp. 14863-14868, 1998.

[17] T. R. Golub, D. K. Slonim, P. Tamayo et al., "Molecular classification of cancer: class discovery and class prediction by gene expression monitoring," Science, vol. 286, no. 5439, pp. 531-527, 1999.

[18] H. Mirzaei, M.-Y. Brusniak, L. N. Mueller, S. Letarte, J. D. Watts, and R. Aebersold, "Halogenated peptides as internal standards (H-PINS): introduction of an MS-based internal standard set for liquid chromatography-mass spectrometry," Molecular and Cellular Proteomics, vol. 8, no. 8, pp. 1934-1946, 2009.

[19] D. Winter, J. Seidler, D. Kugelstadt, B. Derrer, B. Kappes, and W. D. Lehmann, "Minimally permutated peptide analogs as internal standards for relative and absolute quantification of peptides and proteins," Proteomics, vol. 10, no. 7, pp. 1510$1514,2010$.

[20] M. S. Lipton, L. Pǎá-Toli, G. A. Anderson et al., "Global analysis of the Deinococcus radiodurans proteome by using accurate mass tags," Proceedings of the National Academy of Sciences of the United States of America, vol. 99, no. 17, pp. 11049-11054, 2002.

[21] E. F. Strittmatter, P. L. Ferguson, K. Tang, and R. D. Smith, "Proteome analyses using accurate mass and elution time peptide tags with capillary LC time-of-flight mass spectrometry," Journal of the American Society for Mass Spectrometry, vol. 14, no. 9, pp. 980-991, 2003.

[22] R. Fang, D. A. Elias, M. E. Monroe et al., "Differential labelfree quantitative proteomic analysis of Shewanella oneidensis cultured under aerobic and suboxic conditions by accurate mass and time tag approach," Molecular and Cellular Proteomics, vol. 5, no. 4, pp. 714-725, 2006.

[23] E. O. Hochleitner, B. Kastner, T. Fröhlich et al., "Protein stoichiometry of a multiprotein complex, the human spliceosomal U1 small nuclear ribonucleoprotein: absolute quantification using isotope-coded tags and mass spectrometry," Journal of Biological Chemistry, vol. 280, no. 4, pp. 2536-2542, 2005.
[24] G. Wang, W. W. Wu, W. Zeng, C.-L. Chou, and R.-F. Shen, "Label-free protein quantification using LC-coupled ion trap or FT mass spectrometry: reproducibility, linearity, and application with complex proteomes," Journal of Proteome Research, vol. 5, no. 5, pp. 1214-1223, 2006.

[25] Z. Zhu, A. R. Boobis, and R. J. Edwards, "Identification of estrogen-responsive proteins in MCF-7 human breast cancer cells using label-free quantitative proteomics," Proteomics, vol. 8, no. 10, pp. 1987-2005, 2008.

[26] K. Podwojski, A. Fritsch, D. C. Chamrad et al., "Retention time alignment algorithms for LC/MS data must consider nonlinear shifts," Bioinformatics, vol. 25, no. 6, pp. 758-764, 2009.

[27] B. Fischer, J. Grossmann, V. Roth, W. Gruissem, S. Baginsky, and J. M. Buhmann, "Semi-supervised LC/MS alignment for differential proteomics," Bioinformatics, vol. 22, no. 14, pp. e132-e140, 2006. 

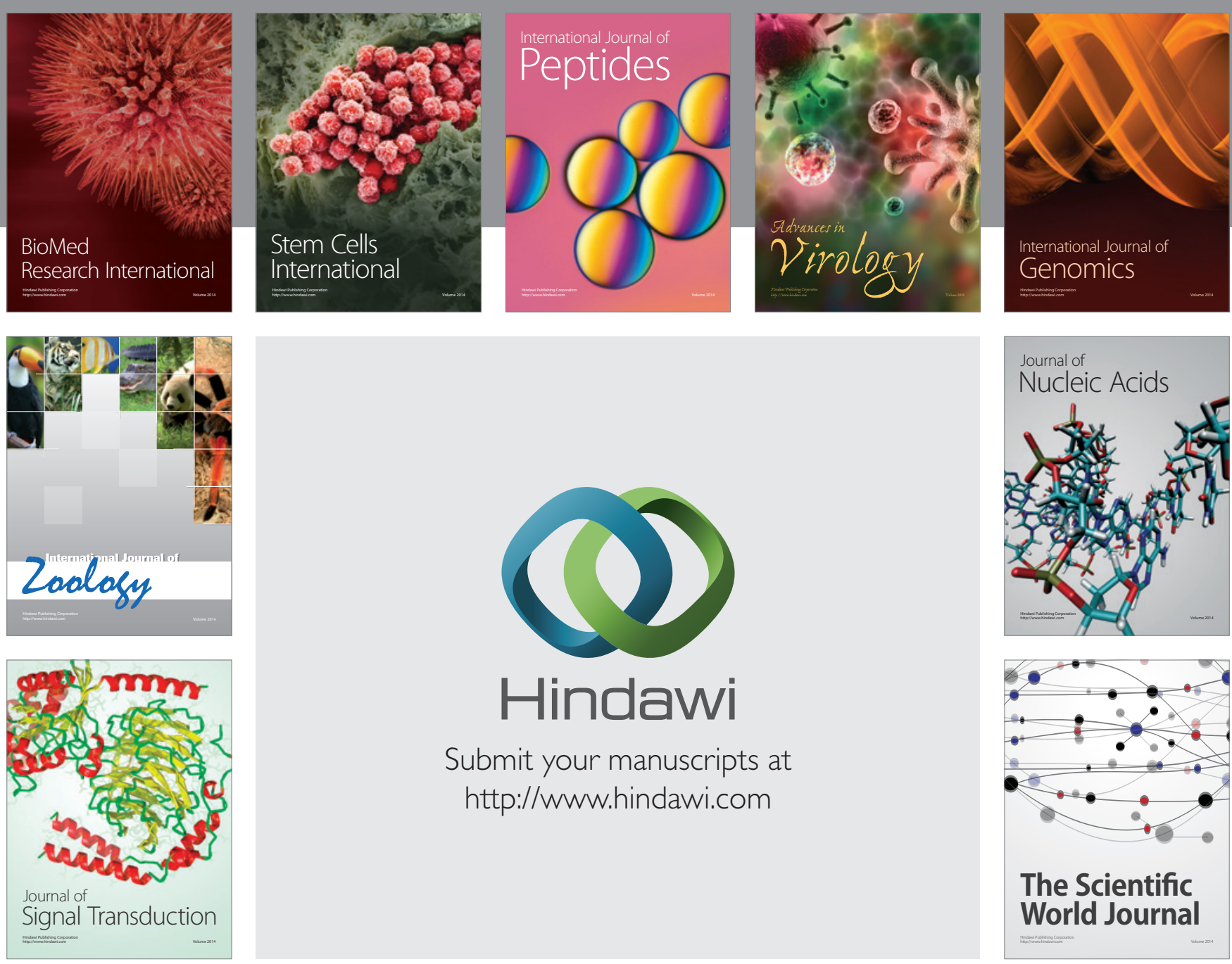

Submit your manuscripts at

http://www.hindawi.com
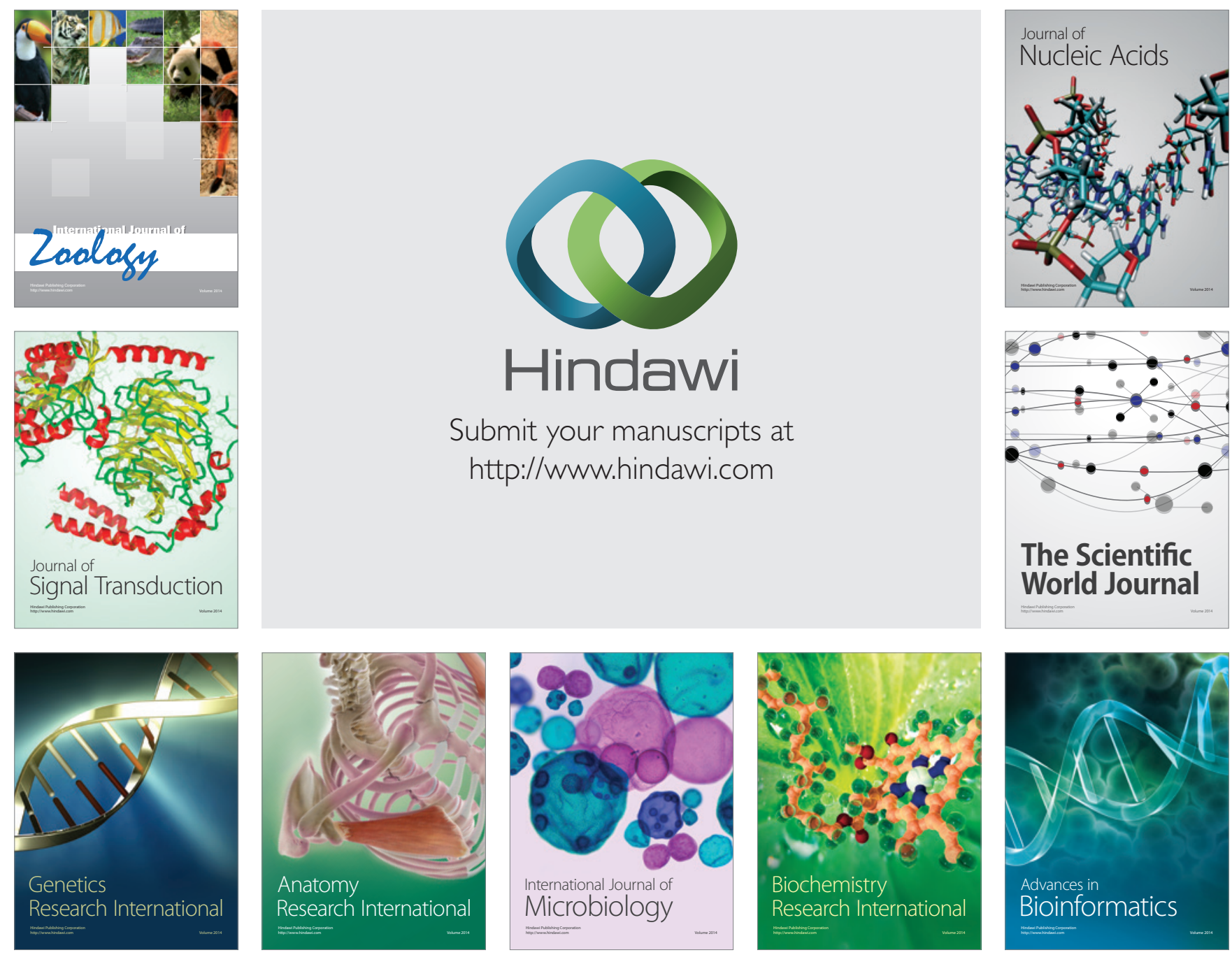

The Scientific World Journal
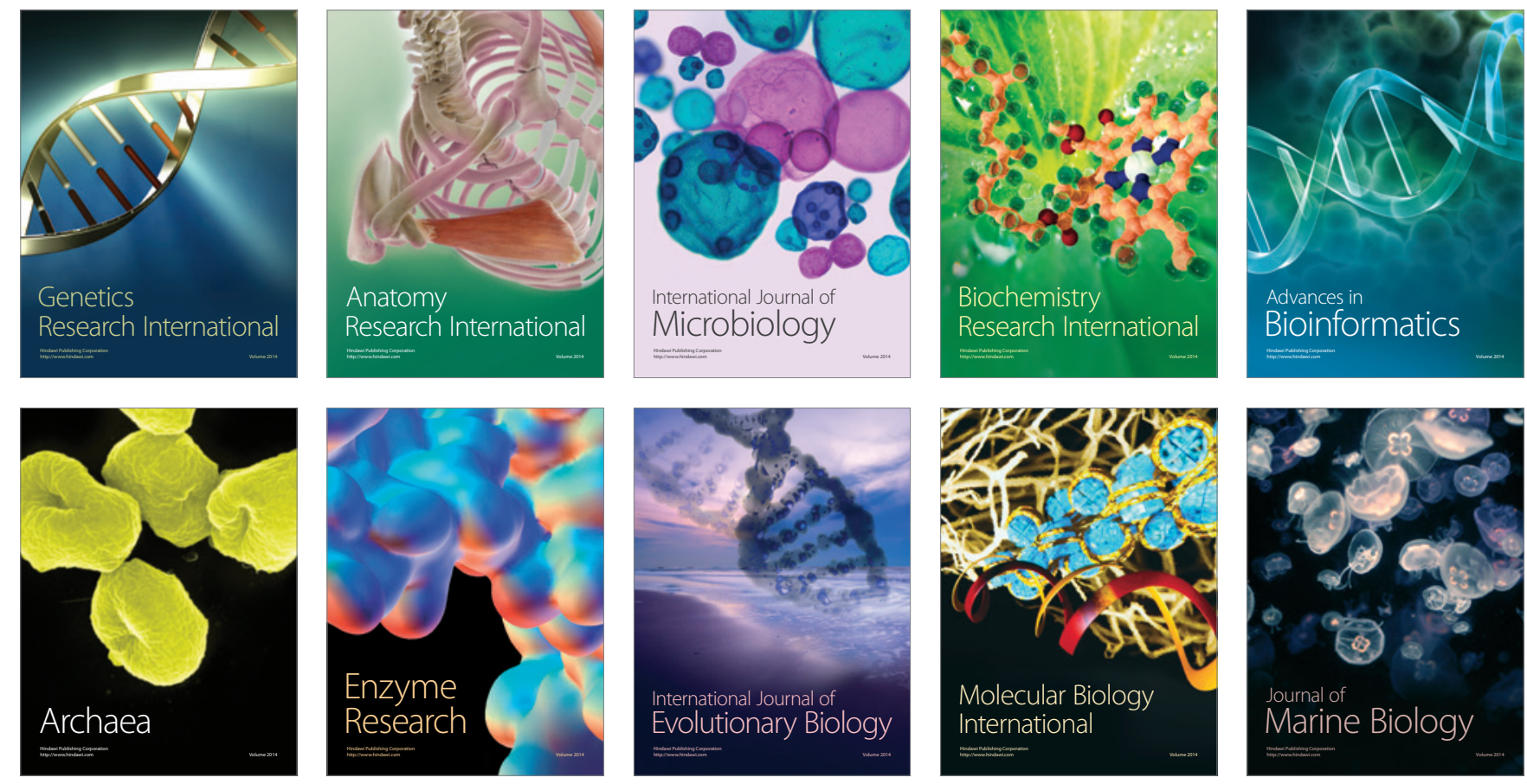